\title{
Electrodynamics of Pulsar Magnetospheres
}

\author{
Anatoly Spitkovsky \\ KIPAC, Stanford University, P.O. Box 20450, MS 29, CA 94309, USA
}

\begin{abstract}
I review the theoretical understanding of the global structure of pulsar magnetospheres concentrating on recent progress in force-free electrodynamics and first-principles simulations of magnetospheres.
\end{abstract}

\section{Introduction}

It is customary to begin reviews of pulsar magnetosphere research by calculating the ratio of the number of outstanding questions about pulsars to the number of years passed since pulsars were discovered. While this ratio probably seems to decrease with time, it does so mainly due to the inevitable growth of the denominator (now at 36), and most of the fundamental questions about pulsars are still with us. This seems to scare researchers away from working on the subject. Yet, in my view, the fact that even basic conceptual questions about pulsars are not fully understood represents an opportunity to learn important insights even from simplified models.

In recent years, the subject has experienced a gradual growth of interest which came with the reluctant realization that the structure of pulsar magnetospheres could not be solved analytically in closed form. This and the development of algorithms applicable to highly magnetized plasma environments brought simulations to the forefront as the alternative tool. I will review the current status of pulsar electrodynamics research concentrating on simulations, and on what they teach us about pulsars and the way we simulate them.

\section{Pulsar Basics}

The fundamentals of pulsar energetics can be understood by considering the spin-down of a rotating conducting magnetized sphere. Two ingredients are needed for its electromagnetic spin-down. First is the existence of a region of rotating magnetic field, which induces electric field $E \sim(\Omega r / c) B$, and the second is the sweepback of magnetic field. This combination results in an outwards Poynting flux, with energy loss rate $\dot{\varepsilon}=\frac{c}{4 \pi} \int(\mathbf{E} \times \mathbf{B}) \cdot d \mathbf{s}$. The integral can be evaluated at a fiducial radius $R_{f}$ where the sweptback toroidal field is equal to the poloidal magnetic field: $\dot{\varepsilon} \sim-\Omega R_{f}^{3} B^{2}\left(R_{f}\right)$. For dipolar field being swept back at the light cylinder $R_{f} \sim R_{L} \equiv c / \Omega$ we get

$$
\dot{\varepsilon} \approx-\frac{\mu^{2} \Omega^{4}}{c^{3}} f(\chi)
$$

where $\mu$ is the magnetic moment, and $f(\chi)$ is a geometric function that depends on the angle $\chi$ between the rotation and magnetic axes. The well-known formula 
for dipole spin-down in vacuum is a special case of $(1)$ with $f_{v a c}(\chi)=2 \sin ^{2} \chi / 3$. The argument leading to (1) also holds for plasma-filled magnetospheres with a wind as long as the outflow is Poynting dominated. The physical reasons for sweepback and electric field in the magnetosphere are different between the vacuum and plasma-filled case. In vacuum the electric field is due to induction while sweepback is due to displacement current, with both effects disappearing for $\chi \rightarrow 0$. The presence of plasma, however, provides electric field due to net charge density in the magnetosphere (e.g., corotation electric field requires GoldreichJulian charge density $\rho_{G J}=-\nabla \cdot(\boldsymbol{\Omega} \times \mathbf{r} \times \mathbf{B}) /(4 \pi c)=-\boldsymbol{\Omega} \cdot \mathbf{B} /(2 \pi c)+$ relativistic corrections). The sweepback of the magnetic field is driven by poloidal currents, with current density $j_{G J}=\rho_{G J} c$ being of the right order of magnitude to provide $B_{\phi}=B_{\text {poloidal }}$ around the light cylinder. Unlike the vacuum case, the presence of plasma leads to spin-down even for aligned rotators, $\chi=0$. How such currents are set up, circulate and close is a central theme of pulsar magnetospheric research. Not surprisingly, the details should depend on the mechanisms of plasma supply, notably on pair creation. In the interest of keeping attention on the global picture, I will consider only two limits: 1) plasma is supplied only from the surface of the star, and 2) plasma is abundant in the magnetosphere. The real pulsar with gaps, pair-formation fronts, etc., lies somewhere in between the two extremes.

\section{Plasma Supply From the Surface}

Rotation of conductors in magnetic fields separates charges inside the conductor due to Lorentz force and leads to potential differences on the surface (unipolar induction). Electric fields outside the conductor develop accelerating components $\left(E_{\|}=\mathbf{E} \cdot \mathbf{B} /|\mathbf{B}|\right)$ which can extract charges from the surface if the work function is sufficiently small. This extraction lead Goldreich \& Julian (1969) to propose a charge-separated model of magnetosphere filled with plasma at particle density equal to $\rho_{G J} / e$. The viability of this model has been questioned over the years (e.g., Holloway 1973), and an unsolved issue of principle is whether an aligned rotator with particle emission only from the surface would spin down (and hence form an active current system).

The evolution of a rotating sphere with negligible work function has been simulated by several groups (Krauss-Polstorff \& Michel 1984, 1985; Smith, Michel \& Thacker, 2001; Petri, Heyvaerts \& Bonazolla 2002a; Spitkovsky \& Arons 2002, and in preparation, hereafter SA). The first three groups used variations of an iterative procedure that constructed axisymmetric $2 \mathrm{D}$ chargeseparated magnetospheres by releasing charges from the surface, finding their equilibrium locations and then iterating locations of other charges until convergence was found. In our work (SA) we use a more general approach by utilizing particle-in-cell (PIC) method for electromagnetic plasma simulation. We represent plasma as a collection of macroparticles and solve inhomogeneous Maxwell equations with currents provided by motion of macroparticles while computing their motion in self-consistent fields. Special care is taken to represent a rotating conducting sphere as a boundary condition. The method is $3 \mathrm{D}$ and is geared towards simulating dynamic situations and not limited to static equilibria.

So, what happens when a conducting sphere (or star) with a dipole magnetic field is rotated? Unipolar induction generates electric field corresponding to a 
central monopolar charge plus quadrupolar surface charge. This field pulls negative charges over the poles $(\boldsymbol{\Omega} \| \mu)$ and positive charges in the equatorial region (Figs. 1[a]-[c]) and has natural trapping regions where $\mathbf{E} \cdot \mathbf{B}=\mathbf{0}$. Once particles start to populate the magnetosphere they provide quadrupolar space charge which tends to cancel the accelerating electric field at the surface, reducing the surface charge and particle injection, approaching a quasi-steady state. This state, reproduced by simulations from all groups, consists of negative "domes" of charge at magnetic poles, and a positive torus around the equator, with electric gap in between. Inside the plasma regions the accelerating electric field is zero, and the plasma density is on the order of, but not exactly, $\rho_{G J}$. The total extent of the charge-separated configuration is several radii of the star, and the magnetosphere is dead: there is no longitudinal current, no magnetic sweepback, and no spin-down.
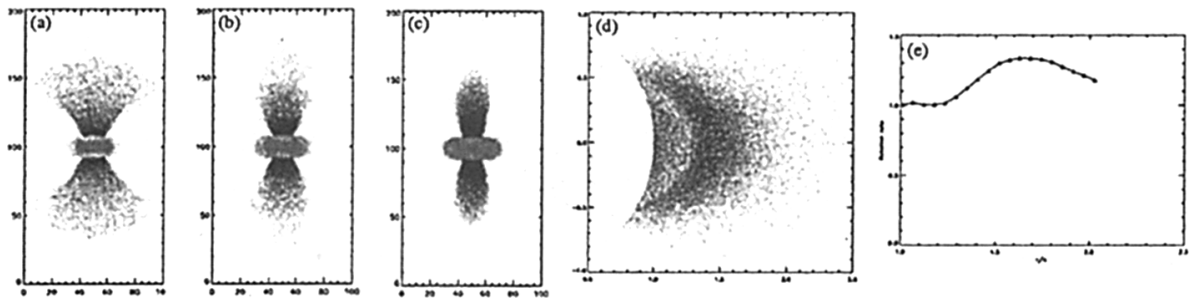

Figure 1. Formation of a charge-separated magnetosphere with particle emission from the surface of a conducting sphere with dipole field.

At least that is the story for axisymmetric simulations. In $3 \mathrm{D}$ the quasistatic configuration is actually unstable. Some field lines that go through the equatorial torus also pass through regions of vacuum gaps. These field lines are not equipotentials and hence plasma on them is not corotating with the star. Figures 1(d) and 1(e) show the cross-section through the positive torus and a plot of the plasma rotation rate (in units of stellar $\Omega$ ) along an equatorial radius. Differential rotation in non-neutral plasma leads to a shear instability known as the diocotron instability. In it, the azimuthal charge density perturbations grow by transporting charges across the field lines. The transport is due to the $\mathbf{E} \times \mathbf{B}$ drift in a perturbed azimuthal electric field and a poloidal magnetic field, and was observed in the simulations of SA. The growth rate for the instability is on the order of several stellar periods, comparable to the linear instability analysis of Petri, Heyvaerts \& Bonazzola (2002b). The instability feeds off the differential rotation of the plasma and tries to establish corotation by moving charge density. The nonlinear simulations (Fig. 2) show the equatorial torus growing with time and approaching the GJ density.

The fact that in 3D the static "dome-disk" electrospheres are unstable and display evolution to corotation suggests that dismissing charge-separated magnetospheres as dead is a bit premature. Having a corotating closed zone is a crucial part of the GJ model, because rotation of charges in the closed zone provides the toroidal current needed to modify and open the poloidal field lines. Radial transport of particles in the equator is a current that changes the charge on the star, and the domes expand to compensate. The present simulations have not yet convincingly addressed the limit when the domes and disk expand out 

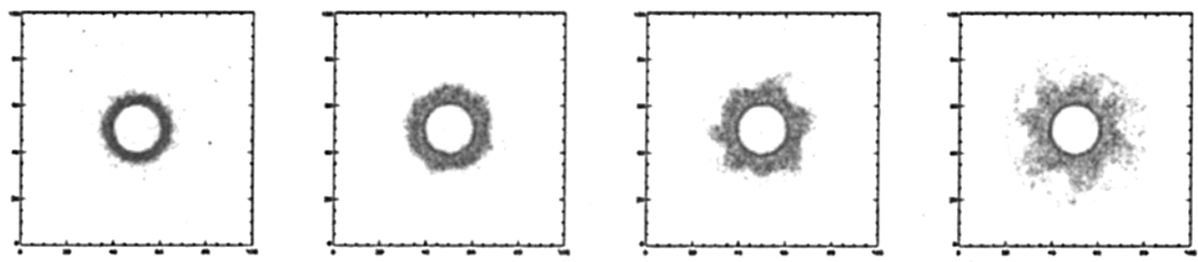

Figure 2. Development of the diocotron instability in the equatorial disk of a charge-separated aligned rotator.

to the light cylinder, when the instability turns from essentially electrostatic to electromagnetic. In the absence of a definitive simulation, I will speculate on a possible scenario that can bring a charge-separated magnetosphere alive. Initial expansion of the corotating closed zone proceeds up to the light cylinder where charge pileup causes regions with $E>B$ to form. The particles decouple from the magnetic field and leave generating the current in the equator. This current connecting through the star causes current flow in the negative domes, perhaps with particles slingshot away in the vicinity of the light cylinder as well. This initial longitudinal current flow modifies the magnetic geometry, causing sweepback, and gradually particles both in the equatorial region and in the domes recouple to the field lines, now with a wind outflow. The diocotron instability and the development of transient accelerating regions is speculatively used here to jump-start the current flow.
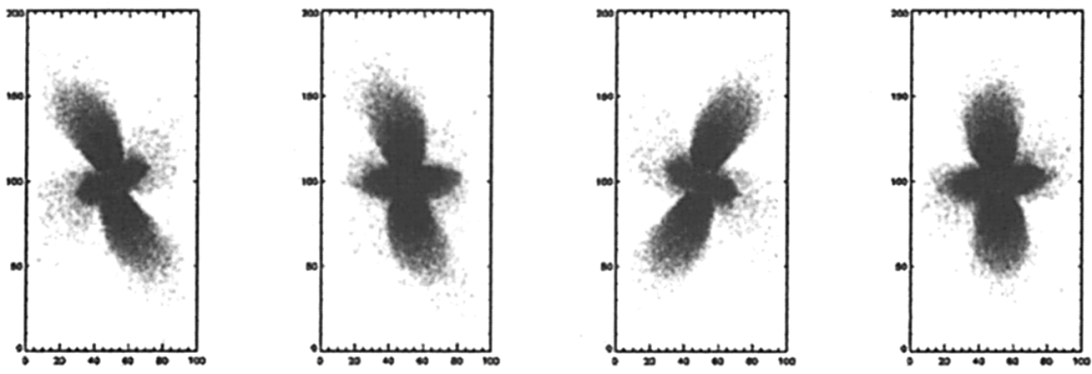

Figure 3. Development of electrosphere and diocotron instability for $30^{\circ}$ oblique rotator: $3 \mathrm{D}$ view.

Another possibility for jump-starting a pulsar, which is still virtually unexplored, is the effect of magnetic inclination. Our preliminary simulations (Fig. 3) show that even for an oblique $\left(30^{\circ}\right)$ rotator, the "dome-disk" electrosphere is formed, albeit with the torus distorted into disjoint halves. The diocotron instability still takes place, and the torus grows. Peculiar to the oblique rotator is the wave pressure from the dipole spin-down, which increases towards $R_{L C}$. The wave pressure initially drives a small fraction of particles away, potentially starting a current flow. The study of long-term evolution of oblique rotators is currently underway. Even if charge-separated magnetospheres turn out to be dead in the end, the charge transport due to the diocotron instability may 
still operate in the closed zone of an active pulsar. The field lines that connect to the star through a null surface will lose corotation if their charge content is perturbed. The diocotron instability may then transport charge across the field lines to return the closed zone to corotation.

\section{Force-Free Magnetospheres}

It is commonly thought that the problems with charge-separated magnetospheres would be readily solved by introducing sufficient amount of quasineutral plasma, most likely due to pair creation. This plasma is able to quickly short out accelerating fields so that the ideal $\mathrm{MHD}$ condition $\mathbf{E} \cdot \mathbf{B}=\mathbf{0}$ holds everywhere. The structure of the magnetosphere is then determined by the equilibrium of forces acting on the plasma. In the limit of small plasma inertia and temperature, the right hand side of the momentum equation becomes $\rho \mathbf{E}+\frac{1}{c} \mathbf{j} \times \mathbf{B}=0$, or "relativistic force-free". Significant effort was directed over the years to finding a steady-state axisymmetric solution for the structure of magnetosphere of an aligned rotator subject to these constraints. Introducing the flux function $\Psi$ such that the poloidal field is $\mathbf{B}_{\mathbf{p}}=\nabla \Psi \times \hat{\phi} / r_{c}$ and writing the corotation electric field as $\mathbf{E}=\frac{r_{c} \Omega}{c} \mathbf{B}_{\mathbf{p}} \times \hat{\phi}$, the force-free constraint can be reduced to:

$$
\left(1-x^{2}\right)\left[\frac{\partial^{2} \Psi}{\partial x^{2}}+\frac{\partial^{2} \Psi}{\partial z^{2}}\right]-\frac{1+x^{2}}{x} \frac{\partial \Psi}{\partial x}=-I(\Psi) I^{\prime}(\Psi) \text {. }
$$

Here, $x \equiv r_{c} / R_{L}$ and $z \equiv z_{c} / R_{L}$ are scaled cylindrical coordinates, and $I(\Psi)$ is an unknown function proportional to the poloidal current enclosed by the flux surface, $\Psi$. Known as the "pulsar equation" (Michel 1973a), Equation (2) is a nonlinear second order elliptic equation with a regular singularity at the light cylinder, $x=1$. The current distribution, $I(\Psi)$, is a priori unknown and should be determined as part of the self-consistent solution. Analytic solutions for the pulsar equation are known only for a monopolar magnetic field (Michel 1973a), and for a corotating dipole magnetosphere without a current (Michel 1973b). A great advance has recently been made by Contopoulos, Kazanas \& Fendt (1999, hereafter CKF) who solved the pulsar equation numerically for dipole magnetic field. They used an elliptic solver to solve Equation (2) both inside and outside the light cylinder for a trial current distribution. In general, such a solution has an unphysical kink in the magnetic field at the light cylinder. A clever iterative algorithm that adjusted the current function to minimize the discontinuity allowed CKF to find a solution that passed smoothly through the light cylinder. This solution, shown in Figure 4(a), has a corotating closed zone and an open wind zone, with poloidal field lines asymptotically becoming monopolar, and a toroidal field dominating at infinity. The last open field line corresponds to $\Psi_{\text {open }}=1.36 \Psi_{p c}$, where $\Psi_{p c} \equiv \mu / R_{L}$ is the flux through the polar cap for an unperturbed dipole field.

The magnetosphere supports an active current system: current is flowing along the open field lines to infinity, and the bulk of return current flows in the equatorial current sheet and on the boundary of closed and open zones, although a $5 \%$ fraction is distributed along a set of open field lines near the equator $\left(1.08 \Psi_{p c}<\Psi<1.36 \Psi_{p c}\right)$. The CKF solution is intuitively pleasing, and should be analyzed further for stability and used as a global model for studying plasma processes in pulsars. However, the questions of validity and uniqueness of the 
CKF solution still linger. At the time of this writing, the CKF result could not be numerically reproduced by several groups. At issue is the representation of a delta-function return current at the closed-open field line interface. CKF reported smoothing this transition with a Gaussian; however, repeating this prescription have not yielded convergence. Instead, Ogura \& Kojima (2003) and the present author found that there exists another smooth solution with $\Psi_{\text {open }}=$ $1.66 \Psi_{p c}$ when the delta-function current is ignored (Fig. 4[b]). Although this is an unphysical solution with $E>B$ beyond the light cylinder, it demonstrates that convergence to a smooth solution is not a guarantee of uniqueness and should be taken with caution.
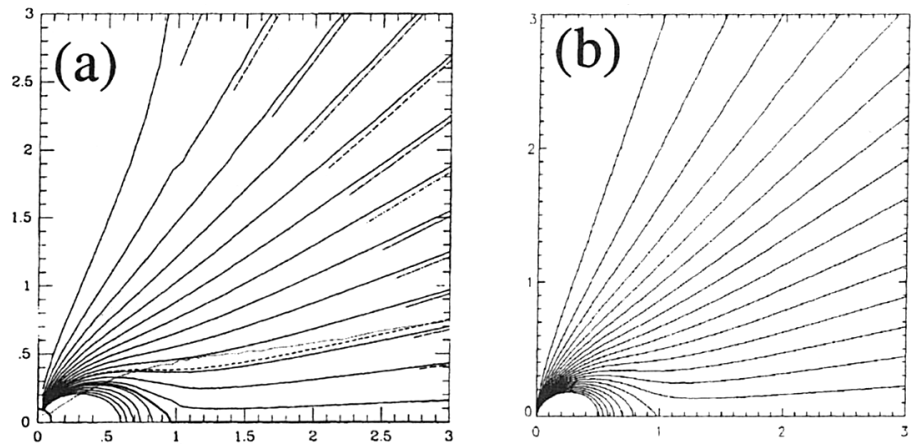

Figure 4. Solutions to the pulsar equation: (a) CKF solution, (b) unphysical but smooth solution without return current.

A number of pulsar magnetosphere models constructed as smooth solutions at the light cylinder encountered regions with $E>B$ further away. Such regions were interpreted as dissipation regions that made current closure via particles crossing the flux surfaces (e.g., Mestel \& Shibata 1994). The CKF solution reports to have $E<B$ throughout, and, especially if verified, serves as a counter-example where force-free electrodynamics holds everywhere in the flow (potentially with the exception of the current sheet regions). Although its existence is not a guarantee that it is stable and will be chosen by a dynamical system, the CKF solution is very attractive on theoretical grounds, and is supported by observational absence of copious amounts of radiation that would emerge from a dissipation layer near $R_{L}$.

\section{Time-Dependent Models}

The questions of stability and uniqueness of magnetospheric solutions are better addressed with a time-dependent simulation than with iterative steady-state searches. It also is tempting to simply set up the appropriate dynamical equations with boundary conditions and let the problem "solve itself". The limit of abundant supply of inertialess plasma with large magnetization is well described by the force-free electrodynamics equations (Blandford 2002):

$$
\begin{gathered}
\frac{1}{c} \frac{\partial \mathbf{E}}{\partial t}=\nabla \times \mathbf{B}-\frac{4 \pi}{c} \mathbf{j} ; \quad \frac{1}{c} \frac{\partial \mathbf{B}}{\partial t}=-\nabla \times \mathbf{E} ; \\
\mathbf{j}=\frac{c}{4 \pi}(\nabla \cdot \mathbf{E}) \frac{\mathbf{E} \times \mathbf{B}}{B^{2}}+\frac{c}{4 \pi} \frac{(\mathbf{B} \cdot \nabla \times \mathbf{B}-\mathbf{E} \cdot \nabla \times \mathbf{E}) \mathbf{B}}{B^{2}} .
\end{gathered}
$$


The first two are just Maxwell's equations, while the third is a current prescription that is derived from the force-free constraint and the ideal condition $\partial_{t}(\mathbf{E} \cdot \mathbf{B})=0$. The two terms in Equation (4) represent the transverse current due to advection of charge density perpendicular to field lines and the longitudinal current, respectively. The system of Equations (3) and (4) is hyperbolic and can be evolved in time. We solved these equations using the finite-difference time-domain (FDTD) method in spherical coordinates with rotating conducting sphere boundary conditions for aligned magnetic fields. Below I describe two test cases that illustrate the nature of force-free time-dependent electrodynamics: a spin-up of a sphere with an initially monopolar magnetic field, and a sphere with a dipole. The Equations (3) and (4) support two wavemodes: fast (electromagnetic) and Alfvén. In general, Alfvén waves have non-zero charge density, $(\mathbf{k} \cdot \mathbf{E})$, and they can transport charge along the magnetic field. Dynamics of these waves allows the magnetosphere to adjust to perturbations. For instance, in Figure 5(a) an Alfvén wave is launched when a sphere with monopolar magnetic field starts rotating. Behind the wave-front, Goldreich-Julian charge and current density are established, making the field lines corotate. The transient wave propagates out to infinity; the simulation reproduces the analytic solution by Michel (1973a) and has no pathology at the light cylinder.
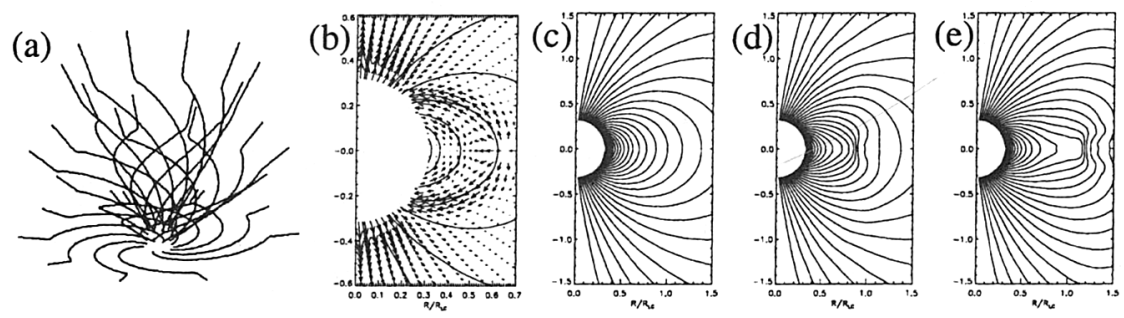

Figure 5. (a) Alfvén wave from a rotating magnetic monopole; (b) Snapshot of formation of return current for a dipole field; (c)-(e) Evolution of poloidal field for a rotating dipole.

The situation is more involved for a rotating dipole. The initial evolution (shown in Fig. 5[b]) produces Alfvén waves which carry outwards current on all field lines (for simplicity $\mu$ and $\boldsymbol{\Omega}$ are counteraligned in this example). Because Alfvén waves follow the field lines, poloidal currents from opposite hemispheres start to cancel each other in the closed zone. As signals from higher latitudes cancel, the closed zone expands outwards, now polarized with the GoldreichJulian charge density. The currents do not cancel at the edge of the closed zone, and this is where a return current sheath develops (Fig. 5[b]). Corotation of the closed zone produces toroidal current, and the structure of the poloidal field lines, shown in Figures 5(c)-(e), starts to change - the field lines are pulled out by the electromagnetic stress, and more flux is passing through the light cylinder compared to the unperturbed dipole (as in the CKF solution). As the field lines are stretched horizontally, the net magnetic field in the equator is decreasing. After $1 / 4$ of a rotational period, the total field in the equator at the light cylinder actually reaches zero, at which point the simulation breaks (note the denominator of Eq. [4]). In the process of opening the field lines, the 
system tries to spontaneously form a current sheet in the equator separating the opposite toroidal and poloidal magnetic fields. Such a current sheet is likely to be non-force-free, and requires the return of resistivity and pressure to be supported. It is still an open question as to whether it is possible to modify Equations (3) and (4) by adding a prescription for resistivity or pressure effects to incorporate current sheets into an otherwise ideal force-free simulation. An interesting conceptual question is whether one can always smoothly pass from one ideal force-free configuration to another without invoking non-ideal effects. A simple example is two CKF magnetospheres of different spin. Continuous transformation between them by changing the spin of the star involves converting some open flux to closed or vice-versa, and hence suggests the need for resistivity. Thus, time-dependent evolution of force-free ideal equations suggests that extra physics needs to be included to establish the steady state solution for the pulsar case (note that this extra physics would likely act only in the current sheet and is different from an extended dissipation zone as in Mestel \& Shibata 1994).

So, where do we go from here? I have outlined several directions of numerical attack on the problem of global structure of pulsar magnetospheres. None have yielded a conclusive answer, although all signs for the aligned rotator with ample plasma supply are pointing to a CKF-type solution. I am optimistic that this solution will eventually get confirmed, or that a similar solution exists. Especially promising is the development of time-dependent global models of the magnetosphere, as they have huge observational implications. It is also the only way to address the problem of oblique rotators. Once the right set of equations with enough physics is postulated, it is a matter of time before it will get solved. One set of equations that certainly has enough physics is relativistic MHD; however, RMHD becomes difficult to solve numerically in the high magnetization (force-free) limit, so some sort of hybrid scheme is probably required.

\section{References}

Blandford, R. D. 2002, in Lighthouses of the Universe, eds. M. Gilfanov et al., (Berlin: Springer), p. 381

Contopoulos, J., Kazanas, D., \& Fendt, C. 1999, ApJ, 511, 351 (CKF)

Goldreich, P., \& Julian, W. H. 1969, ApJ, 160, 971

Holloway, N. J. 1973, Nature Phys. Sci., 246, 6

Krause-Polstorff, J., \& Michel, F. C. 1984, MNRAS, 213, 43

Krause-Polstorff, J., \& Michel, F. C. 1985, A\&A, 144, 72

Mestel, L., \& Shibata, S. 1994, MNRAS, 271, 621

Michel, F. C. 1973a, ApJ, 180, L133

Michel, F. C. 1973b, ApJ, 180, 207

Ogura, J., \& Kojima, Y. 2003, Prog. Theor. Phys., 109, 619

Petri J., Heyvaerts J., \& Bonazzola, S. 2002a, A\&A, 384, 414

Petri J., Heyvaerts J., \& Bonazzola, S. 2002b, A\&A, 387, 520

Smith, I. A., Michel, F. C., \& Thacker, P. D. 2001, MNRAS, 322, 209

Spitkovsky, A., \& Arons, J. 2002, in ASP Conf. Series, Vol. 271, Neutron Stars in Supernova Remnants, eds. P. O. Slane, \& B. M. Gaensler, (San Francisco: ASP), p. 81 (SA) 